\title{
População Folicular Ovariana Durante o Ciclo Estral em Vacas da Raça Gir ${ }^{1}$
}

\section{Ademir de Moraes Ferreira ${ }^{2}$, João Henrique Moreira Viana², Luiz Sérgio de Almeida Camargo², Wanderlei Ferreira de Sá2 ${ }^{\text {, Pedro Augusto Carvalho Pereira }}{ }^{3}$}

\footnotetext{
RESUMO - A população folicular ovariana de 14 vacas da raça Gir foi monitorada diariamente, por ultra-sonografia, ao longo de um ciclo estral completo. Em cada avaliação, os folículos ovarianos foram identificados, mensurados e classificados por tamanho, em três categorias: de 3 a $5 \mathrm{~mm}$ (folículos pequenos), de 6 a $8 \mathrm{~mm}$ (folículos médios) e iguais ou superiores a $9 \mathrm{~mm}$ (folículos grandes). Foram observados ciclos com três $(69,23 \%)$ e quatro $(30,77 \%)$ ondas de crescimento folicular. O número médio de folículos presentes ao longo do ciclo estral foi de $12,85 \pm 0,26$, tendo a população de pequenos folículos constituído a maior fração deste total $(70,08 \%$ a $93,18 \%)$. Observou-se efeito significativo do dia do ciclo sobre o número de folículos identificados em cada classe de tamanho. A população de folículos pequenos e a população folicular total apresentaram redução significativa ao longo do ciclo. A população de folículos médios foi maior nos animais que apresentaram ciclos com quatro ondas de crescimento folicular. A população folicular total e o número de folículos de cada classe de tamanho nos ovários variaram ao longo do ciclo estral em decorrência do estabelecimento de ondas de crescimento folicular.
}

Palavras-chave: bovinos, dinâmica folicular, ultra-som

\section{Ovarian Follicular Population During Estrous Cycle in Gir Breed (Bos indicus) Cows}

\begin{abstract}
The aim of this study was to evaluate follicular population during estrous cycle in Gir cattle. The ovaries of cows (14) were evaluated daily, during one estrous cycle, using a portable ultrasound device. During each exam, ovarian follicles were identified, measured and classified by size in tree groups: from 3 to $5 \mathrm{~mm}$ (small follicles), from 6 to $8 \mathrm{~mm}$ (medium follicles) and equal or larger than $9 \mathrm{~mm}$ (large follicles). Cycles with three (69.23\%) and four (30.77\%) follicular waves were observed. The mean number of follicles present during the estrous cycle was $12.85 \pm 0.26$. The major part of this population ( $70.08 \%$ to $93.18 \%$ ) was of small follicles. There was a significant effect of estrous cycle day on the number of follicles in each size class. The number of small follicles and the total follicular population decreased during estrous cycle. Cows with four follicular waves during the estrous cycle presented more medium follicles. Total follicular population, and the number of follicles in each size class varied during the estrous cycle according to the establishment of the follicular waves.
\end{abstract}

Key Words: bovine, follicular dynamic, ultrasound

\section{Introdução}

A fêmea bovina apresenta, ao nascimento, em torno de 100.000 folículos primordiais nos ovários. Os folículos que constituem este pool de reserva podem permanecer em um estado quiescente, ou iniciar um processo irreversível de desenvolvimento e que culmina com a ovulação ou, na maior parte das vezes, com a atresia (Findlay, 1994; Erickson \& Shimasaki, 2001). O número de folículos em fase antral, em um determinado momento do ciclo estral, depende da taxa de mobilização da reserva de folículos primordiais e, posteriormente, do suporte proporcionado por fatores de crescimento e gonadotrofinas hipofisárias (Crowe, 1999).
Técnicas de reprodução assistida, como a superovulação e a fertilização in vitro, têm objetivado a obtenção de embriões a partir de oócitos contidos em folículos inicialmente destinados à atresia (Armstrong, 1993; Kruip et al., 1994). Os fatores que controlam o desenvolvimento folicular na fase pré-antral, porém, são pouco conhecidos (Hirshfield, 1994; Picton, 2001), e a eficiência dessas técnicas depende da população de folículos já em fase antral presentes nos ovários (Adams, 1994; Boni et al., 1997). Os oócitos bovinos adquirem competência para o desenvolvimento in vitro quando o folículo atinge de 2 a $3 \mathrm{~mm}$ (Hyttel et al., 1997), limite mínimo para o desenvolvimento em resposta à superovulação, e coincide com o limite de resolução dos equipamentos de ultra-sonografia disponíveis.

\footnotetext{
1 Projeto financiado pela FAPEMIG.

2 Pesquisador, Embrapa Gado de Leite. Rua Eugênio do Nascimento, 610, Juiz de Fora, MG, CEP: $36038-330$.

E.mail: ademirmf@cnpgl.embrapa.br; jhmviana@cnpgl.embrapa.br; camargo@cnpgl.embrapa.br; wandefsa@cnpgl.embrapa.br

${ }^{3}$ Médico Veterinário, Pós-graduando da Escola de Veterinária da UFMG, Belo Horizonte, MG. E.mail: pedrovet9@hotmail.com
} 
O Brasil, atualmente, destaca-se pelo uso de biotecnologias reprodutivas em bovinos, respondendo por, aproximadamente, $13 \%$ dos embriões transferidos no mundo (Thibier, 2001). Apesar da inexistência de números precisos, sua participação na produção in vitro (PIV) de embriões também é considerada expressiva. Os zebuínos têm especial importância neste contexto, e existe crescente necessidade de adaptação de protocolos de produção in vivo e in vitro de embriões para essas raças. O foco inicial dos trabalhos sobre dinâmica folicular, tanto em taurinos como em zebuínos, foi o estabelecimento do número de ondas de crescimento folicular ao longo do ciclo e, posteriormente, o padrão de estabelecimento dos folículos dominantes. Poucos trabalhos descrevem as variações na população folicular ovariana durante o ciclo estral (Pierson \& Ginther, 1987; Savio et al., 1988), particularmente em zebuínos.

Objetivou-se, com este trabalho, caracterizar o número de folículos em fase antral identificáveis por ultra-sonografia ao longo do ciclo estral e, conseqüentemente, disponíveis para produção de embriões em vacas da raça Gir.

\section{Material e Métodos}

Foram utilizadas 14 vacas da raça Gir, pertencentes à Embrapa Gado de Leite, mantidas no Campo Experimental Fazenda Santa Mônica, município de Valença, RJ. Todos os animais apresentavam ciclos estrais regulares e foram previamente submetidos a exames clínico e ginecológico, sendo usados aqueles sem qualquer alteração clínica ou reprodutiva. Os animais receberam suplementação volumosa, constituída de silagem de milho, para manutenção da condição corporal inicial ou obtenção de balanço energético positivo durante o período experimental.

A população folicular ovariana foi monitorada diariamente, ao longo de um ciclo estral completo. $\mathrm{O}$ início do ciclo (dia 0 ) foi estabelecido pela constatação da ovulação subseqüente à observação da manifestação de estro. As avaliações foram realizadas utilizando-se um aparelho portátil de ultra-som ${ }^{1}$, equipado com um transdutor linear bifreqüencial de 5/7,5 MHz. Em cada avaliação, os folículos ovarianos foram identificados e o diâmetro $(\mathrm{mm})$ determinado por meio de dispositivos do aparelho de ultra-som, e classificados por tamanho em três categorias: de 3 a $5 \mathrm{~mm}$ (folículos pequenos), de 6 a $8 \mathrm{~mm}$ (folículos médios) e iguais ou superiores a $9 \mathrm{~mm}$ (folículos grandes). O número de folículos em cada classe de tamanho foi registrado para cada dia do ciclo. A dinâmica folicular foi determinada pelo monitoramento do crescimento e regressão folicular. O número de folículos em emergência, em cada onda, foi caracterizado como o número de folículos que iniciaram seu desenvolvimento simultaneamente, e atingiram um diâmetro mínimo de $5 \mathrm{~mm}$.

O efeito do estádio do ciclo e do número de ondas de crescimento folicular sobre o total de folículos presentes e sobre o número de folículos em cada classe de tamanho foi determinado por análise de variância, enquanto as diferenças de médias, pelo teste Tukey. A variação diária no número de folículos foi avaliada por regressão linear. A relação entre o tamanho médio do maior folículo e o número de folículos foi determinada pelo método das correlações de Pearson. As análises estatísticas foram realizadas por intermédio do programa SAEG 7.1. Os resultados são apresentados na forma de média mais ou menos erro-padrão da média (E.P.M.).

\section{Resultados e Discussão}

Foram observados ciclos com três $(69,23 \%)$ e quatro $(30,77 \%)$ ondas de crescimento folicular. As principais características da dinâmica folicular nos ciclos avaliados são apresentadas na Tabela 1. O número médio diário de folículos identificáveis por ultra-sonografia nos ovários, ao longo do ciclo estral, foi de $13,82 \pm 0,12$ e de $12,71 \pm 0,11$, respectivamente, nos ciclos com quatro e três ondas de crescimento, observando-se a maior média no quarto dia do ciclo, independentemente do número de ondas $(14,93 \pm 0,39)$. Neste dia, o número de folículos presentes variou de 6 , no animal com menor média de folículos em crescimento ao longo do ciclo $(8,58 \pm 0,15)$, a 26 , no animal com maior média $(16,91 \pm 0,20)$, caracterizando grande diferença individual no recrutamento folicular.

O número de folículos presentes no animal com maior média foi superior ao do animal com menor média durante todo o ciclo, sendo a maior diferença absoluta também observada no quarto dia. Coerentemente, os maiores valores para o desvio-padrão da

1 Concept LC, Dynamic Imaging, Scotland.

R. Bras. Zootec., v.33, n.6, p.1689-1694, 2004 (Supl. 1) 
Tabela 1 - Características da dinâmica folicular em vacas da raça Gir apresentando três ou quatro ondas de crescimento folicular durante o ciclo estral

Table 1 - Follicular dynamics characteristics in Gir breed cows presenting three or four follicular waves during estrous cycle

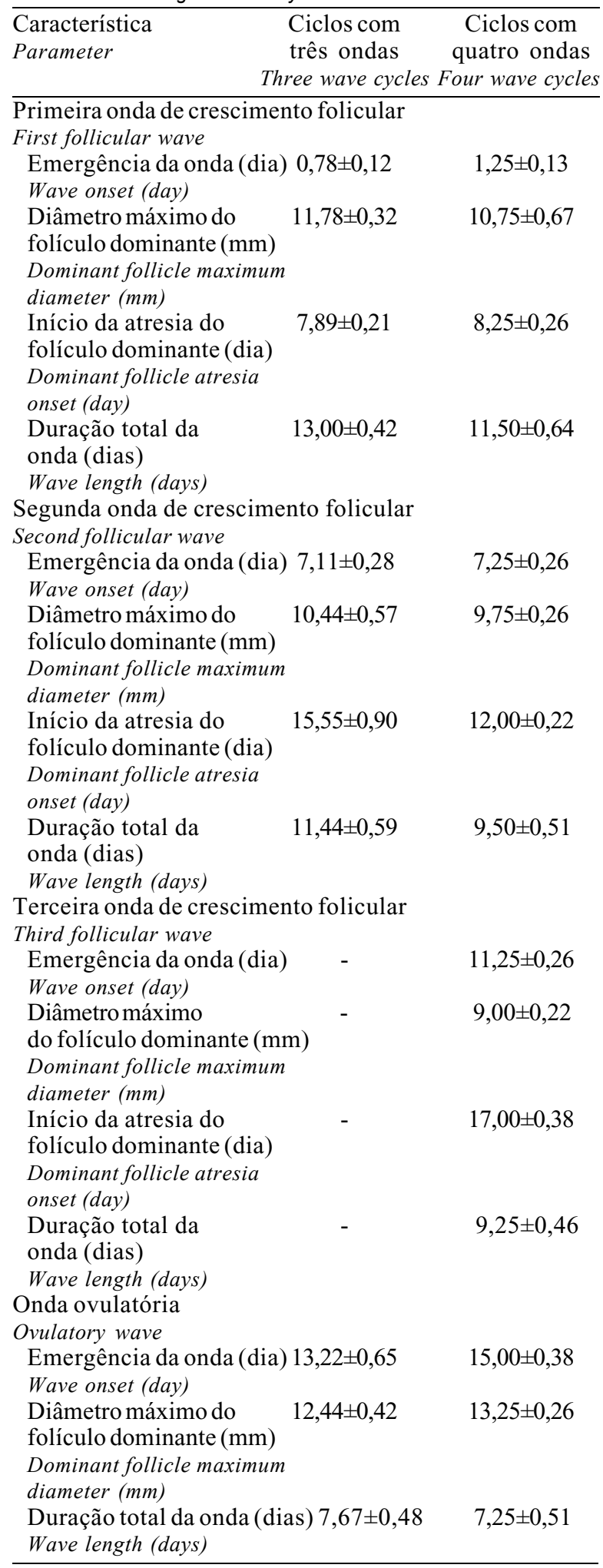

média geral também foram observados entre os dias quatro e cinco (5,43 e 5,58; respectivamente). A primeira onda de crescimento folicular tem sido associada ao recrutamento de maior número de folículos (Pierson \& Ginther, 1987), em razão do possível efeito da liberação de FSH associada ao pico ovulatório de LH (Adams et al., 1992). A emergência da segunda onda de crescimento folicular (Tabela 1) ocorreu no mesmo período em ciclos com três e quatro ondas (7,11 $\pm 1,05$ vs. $7,25 \pm 0,96$, respectivamente; $\mathrm{P}>0,05)$.

$\mathrm{O}$ número de folículos presentes nos ovários é uma das principais fontes de variação nos resultados da superovulação (Viana et al., 1999), e o recrutamento folicular tem sido sugerido como critério de seleção para doadoras de oócitos e embriões (Boni et al., 1997).

A população de folículos pequenos (3 a $5 \mathrm{~mm}$ ) constituiu a maior fração do total de folículos presentes (de 70,08 a 93,18\%, em média). Observou-se efeito significativo $(\mathrm{P}<0,05)$ do estádio do ciclo sobre o número de folículos identificados em cada classe de tamanho (Figura 1). O número de folículos de tamanhos médio ( 6 a $8 \mathrm{~mm}$ ) ou grande $(>8 \mathrm{~mm})$ oscilou ao longo do ciclo, de maneira coerente com o esperado em função do padrão de crescimento folicular em ondas, caracterizado tanto em bovinos de raças taurinas (Knopf et al., 1989; Taylor \& Rajamahendran, 1991) como em zebuínas (Rhodes et al., 1995; Zeitoun et al., 1996; Barros et al., 1998). Com a alternância

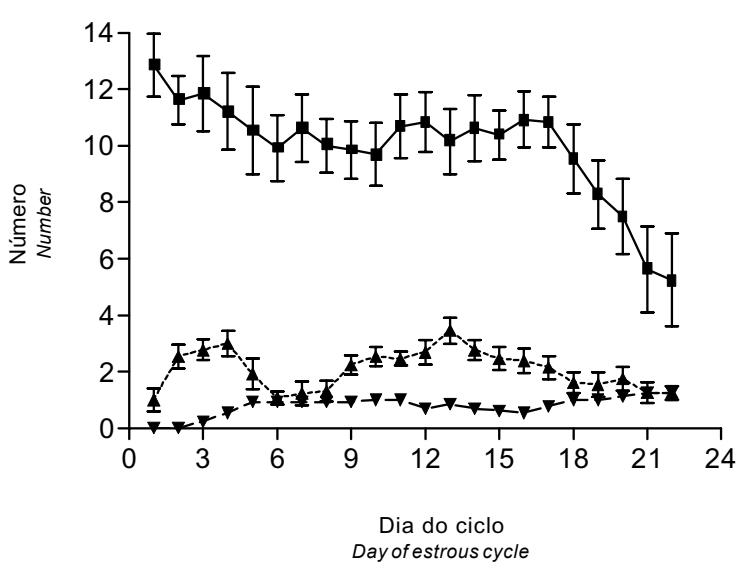

Figura 1 - População folicular ao longo do ciclo estral em vacas da raça Gir. Folículos com diâmetro de 3 a $5 \mathrm{~mm}$ $(n)$, de 6 a $8 \mathrm{~mm}(s)$, ou maiores que $9 \mathrm{~mm}(t)$.

Figure 1 - Follicular population during the estrous cycle in Gir breed (Bos indicus) cows. Follicles with diameter ranging from 3 to $5 \mathrm{~mm}(\mathrm{n}), 6$ to $8 \mathrm{~mm}(\mathrm{~s})$, and above $9 \mathrm{~mm}(t)$. 
das ondas de crescimento folicular, após a atresia do primeiro folículo dominante, observou-se redução da variação na população de folículos médios e grandes, em decorrência da presença simultânea, em determinado dia, de folículos em crescimento e atresia.

A população de folículos médios foi maior $(\mathrm{P}<0,05)$ nos animais que apresentaram ciclos com quatro ondas de crescimento (Tabela 2). Entretanto, não houve diferença $(\mathrm{P}>0,05)$ do número de folículos pequenos ou grandes entre ciclos com três ou quatro ondas de crescimento folicular. A diferença no número de folículos médios pode ser decorrente da menor persistência dos folículos dominantes, observada em ciclos com maior número de ondas (Tabela 1), e de conseqüente redução no intervalo da emergência das ondas. A população de folículos pequenos e, conseqüentemente, a população folicular total apresentaram redução significativa ao longo do ciclo $(\mathrm{P}<0,01)$. Este resultado é semelhante ao relatado por Savio et al. (1988), porém contrastante com aquele obtido por Pierson \& Ginther (1987), em novilhas da raça Holandesa, que verificaram aumento no número total de folículos próximo ao dia da ovulação. Esta variação pode estar relacionada a diferenças no número de ondas de crescimento folicular observadas entre os trabalhos e à maior participação de folículos de diâmetro superior a $5 \mathrm{~mm}$ no número total de folículos em animais de raças taurinas (Pierson \& Ginther, 1987). O número de folículos pequenos $(\mathrm{Y})$ variou em função do dia $(\mathrm{X})$, segundo o modelo $\mathrm{Y}=11,77-0,18 \mathrm{X}\left(\mathrm{R}^{2}=0,31\right)$. A

Tabela 2 - Número de folículos (por classe de tamanho) ao longo do ciclo estral em vacas que apresentaram três ou quatro ondas de crescimento folicular (média \pm E.P.M.)

Table 2 - Follicular population (according to size class) during the estrous cycle in cows presenting three or four follicular waves (mean \pm S.E.M)

Classe de tamanho Ciclos com três Ciclos com quatro $\begin{array}{ccc}\text { Size class } & \text { ondas } & \text { ondas } \\ & \text { Three wave cycles } & \text { Four wave cycles }\end{array}$

Pequenos (3 a $5 \mathrm{~mm}$ ) $10,53 \pm 0,48^{\text {a }} \quad 10,35 \pm 0,41^{\mathrm{a}}$

Small (3 to $5 \mathrm{~mm})$

Médios $(6$ a $8 \mathrm{~mm}) \quad 2,00 \pm 0,16^{\mathrm{a}} \quad 2,51 \pm 0,18^{\mathrm{b}}$

Medium $(6$ to $8 \mathrm{~mm})$

Grandes $(\geq 9 \mathrm{~mm}) \quad 0,76 \pm 0,06^{\mathrm{a}} \quad 0,64 \pm 0,06^{\mathrm{a}}$

Large $(\geq 9 \mathrm{~mm})$

Valores sobrescritos por letras iguais na mesma linha não diferem entre si $(P>0,05)$.

Values superscript by similar letters at the same line do not differ $(P>.05)$. redução na população folicular foi evidente durante os períodos inicial (do $4 \stackrel{0}{\circ}$ ao $6^{\circ}$ dia) e final (após o $16^{\circ}$ dia) do ciclo, que coincidem com o período do estabelecimento do primeiro folículo dominante, e do folículo ovulatório (Tabela 1).

O estabelecimento de um folículo dominante funcional está associado à redução na população folicular presente nos ovários (Bungartz \& Niemann, 1994). O folículo dominante, pela secreção de inibina e estradiol, induz a atresia dos folículos subordinados e bloqueia o recrutamento (Ginther et al., 1996). O folículo dominante é funcional apenas em sua fase de crescimento ou início de estabilização (Adams et al., 1993), e seu efeito sobre a população folicular está relacionado com a duração de seu crescimento (função do tamanho máximo atingido) e com sua persistência. Observaram-se correlações negativas significativas $(\mathrm{P}<0,0001)$ entre o diâmetro do maior folículo ao longo do ciclo e o número de folículos pequenos, médios e total (Tabela 3), independentemente do número de ondas de crescimento observadas.

$\mathrm{O}$ valor destas correlações pode ter sido limitado pela presença de folículos dominantes não-funcionais. $\mathrm{O}$ diâmetro do maior folículo foi menor no período correspondente à segunda onda de crescimento e também à terceira, em ciclos de quatro ondas (Figura 2). $\mathrm{O}$ folículo dominante da segunda onda de crescimento apresenta menor diâmetro final, quando comparado ao da primeira ou ao folículo ovulatório (Ginther et al., 1989; Taylor \& Rajamahendran, 1991).

Tabela 3 - Correlação do número de folículos ao longo do ciclo estral com o diâmetro do maior folículo presente (média \pm E.P.M.)

Table 3 - Correlation coefficient between the number of follicles during estrous cycle and the diameter of the largest follicle present (mean \pm S.E.M.)

\begin{tabular}{lcc}
\hline $\begin{array}{l}\text { Classe de tamanho } \\
\text { Size class }\end{array}$ & $\begin{array}{c}\text { Correlação } \\
\text { Correlation }\end{array}$ & $\begin{array}{c}\text { Significância } \\
\text { Significance }\end{array}$ \\
\hline $\begin{array}{l}\text { Pequenos (3 a } 5 \mathrm{~mm}) \\
\text { Small }(3 \text { to } 5 \mathrm{~mm})\end{array}$ & $-0,34$ & 0,0001 \\
$\begin{array}{l}\text { Médios }(6 \text { a } 8 \mathrm{~mm}) \\
\text { Medium }(6 \text { to } 8 \mathrm{~mm})\end{array}$ & $-0,24$ & 0,0001 \\
$\begin{array}{l}\text { População folicular total } \\
\text { Total follicular population }\end{array}$ & $-0,32$ & 0,0001 \\
\hline
\end{tabular}

R. Bras. Zootec., v.33, n.6, p.1689-1694, 2004 (Supl. 1) 


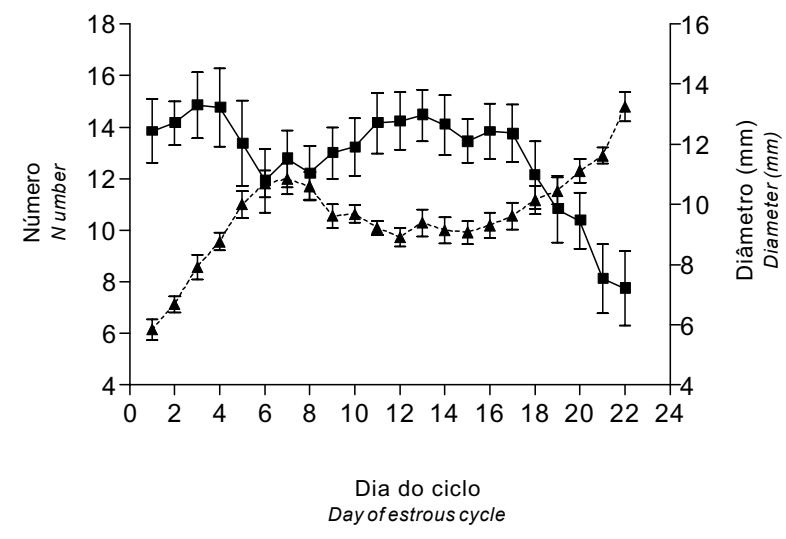

Figura 2 - Número total de folículos $(n)$ e diâmetro do maior folículo presente $(s)$ durante o ciclo estral em vacas da raça Gir.

Figure 2 - Total follicular number $(n)$ and diameter of the largest follicle (s) present during estrous cycles of Gir breed cows (Bos indicus).

Cada onda de crescimento folicular é caracterizada pelo recrutamento e emergência de um grupo de folículos provenientes do pool de folículos em início de fase antral (Fortune, 1994). O recrutamento folicular está associado às elevações transitórias na concentração plasmática de FSH que precedem cada onda de crescimento (Ginther et al., 1996). O número de folículos recrutados e que emergem em cada onda, porém não é constante (Knopf et al., 1989). A redução observada no presente trabalho no número total de folículos poderia estar associada à diminuição normalmente observada no número de folículos emergindo em cada onda de crescimento (Boni et al., 1997). Não houve diferença do número de folículos em emergência entre ondas, ou entre animais que apresentaram três ou quatro ondas de crescimento folicular ( $\mathrm{P}>0,05)$. Observou-se, contudo, tendência numérica de redução no número de folículos emergindo ao longo do ciclo (Tabela 4). O critério utilizado na determinação do número de folículos em cada onda (folículos que iniciaram o crescimento e atingiram diâmetro mínimo de $5 \mathrm{~mm}$ ) evitou o confundimento com folículos de ondas anteriores em atresia, porém reduziu a sensibilidade para detectar eventual redução no recrutamento de folículos pequenos.
Tabela 4 - Número de folículos que iniciaram o crescimento simultaneamente, em cada onda, e atingiram diâmetro mínimo de $5 \mathrm{~mm}$

Table 4 - Number of follicles emerging simultaneously, in each wave, and raising a minimum diameter of $5 \mathrm{~mm}$

Onda de crescimento Média \pm E.P.M. CV folicular Mean \pm S.E.M.

Follicular wave

\begin{tabular}{lcc}
\hline Primeira & $7,21 \pm 0,21^{\text {a }}$ & 40,39 \\
$\begin{array}{l}\text { First } \\
\text { Intermediárias * }\end{array}$ & $5,65 \pm 0,13^{\text {a }}$ & 38,56 \\
$\begin{array}{l}\text { Intermediate } \\
\text { Ovulatória } \\
\text { Ovulatory }\end{array}$ & $4,21 \pm 0,08^{\text {a }}$ & 28,20 \\
Valo & &
\end{tabular}

Valores seguidos por letras iguais na mesma coluna não diferem entre si $(P>0,05)$.

* Segunda, em ciclos de três ondas, e associação da segunda e terceira, em ciclos de quatro ondas.

Values superscript by similarletters at the same column do not differ $(P>05)$.

* Second, in three wave cycles; and association of the second and third, in four wave cycles.

\section{Conclusões}

O número de folículos de cada classe de tamanho e, conseqüentemente, a população folicular total presente nos ovários de vacas da raça Gir variam ao longo do ciclo estral, sendo o número máximo de folículos disponíveis observado no quarto dia do ciclo.

Apesar de ser influenciada pelo padrão de crescimento e pela regressão dos folículos dominantes, a principal fonte de variação no número de folículos presentes é o padrão individual de recrutamento folicular, particularmente durante o período correspondente à primeira onda de crescimento folicular.

\section{Literatura Citada}

ADAMS, G.P.; MATTERI, R.L.; KASTELIC, J.P. et al. Association between surges of follicle-stimulating hormone and the emergence of follicular waves in heifers. Journal of Reproduction and Fertility, v.94, p.177-188, 1992.

ADAMS, G.P.; KOT, K.; SMITH, C.A. et al. Effect of a dominant follicle on regression of its subordinates in heifers. Canadian Journal of Animal Science, v.73, p.267-275, 1993.

ADAMS, G.P. Control of ovarian follicular wave dynamics in cattle: implication for syncronization \& superstimulation. Theriogenology, v.41, n.1, p.19-24, 1994.

ARMSTRONG, D.T. Recent advances in superovulation of cattle. Theriogenology, v.39, n.1, p.7-24, 1993.

BARROS, C.M.; MOREIRA, M.B.P.; FERNANDES, P. Pharmacological manipulation of the estrous cycle to improve artificial insemination or embryo transfer programs. Arquivo da Faculdade de Veterinária da UFRGS, v.26, n.1, p.179-198, 1998 . 
BONI, R.; ROELOFSEN, M.W.M.; PIETERSE, M.C. et al. Follicular dynamics, repeatability and predictability of follicular recruitment in cows undergoing repeated follicular puncture. Theriogenology, v.48, n.2, p.277-289, 1997.

BUNGARTZ, L.; NIEMANN, H. Assessment of the presence of a dominant follicle and selection of dairy cows suitable for superovulation by a single ultrasound examination. Journal of Reproduction and Fertility, v.101, n.3, p.583-591, 1994.

CROWE, M.A. Gonadotrophic control of terminal follicular growth in cattle. Reproduction Domestic Animals, v.34, n.3, p.157-166, 1999.

ERICKSON, G.F.; SHIMASAKI, S. The physiology of folliculogenesis: the role of novel growth factors. Fertility and Sterility, v.76, n.5, p.943-949, 2001

FINDLAY, J.K. Peripheral and local regulators of folliculogenesis. Reproduction Fertility Development, v.6, p.127-139, 1994

FORTUNE, J.E. Ovarian follicular growth and development in mammals. Biology of Reproduction, v.50, n.2, p.225-232, 1994

GINTHER, O.J.; KNOPF, L.; KASTELIC, J.P. Temporal association among ovarian events in cattle during oestrous cycles with two and three follicular waves. Journal of Reproduction and Fertility, v.87, n.1, p.223-230, 1989.

GINTHER, O.J.; WILTBANK, M.C.; FRICKE, P.M. et al. Selection of the dominant follicle in cattle. Biology of Reproduction, v.55, n.6, p.1187-1194, 1996.

HIRSHFIELD, A.N. Relationship between the supply of primordial follicles and the onset of follicular growth in rats. Biology of Reproduction, v.50, n.2, p.421-428, 1994.

HYTTEL, P.; FAIR, T.; CALLESEN, H.; GREVE, T. Oocyte growth, capacitation and final maturation in cattle. Theriogenology, v.47, p.23-32, 1997.

KNOPF,L.; KASTELIC, J.P.; SCHALLENBERGER, E. et al. Ovarian follicular dynamics in heifers: test of two wave hypothesis by ultrasonically monitoring individual follicles. Domestic Animal Endocrinology, v.6, n.2, p.111-119, 1989.

KRUIP, Th.A.M.; BONI, R.; WURTH, Y.A. et al. Potential use of ovum pick-up for embryo production and breeding in cattle. Theriogenology, v.42, n.4, p.675-684, 1994.
PICTON, H.M. Activation of follicle development: the primordial follicle. Theriogenology, v.55, p.1193-1210, 2001.

PIERSON, R.A.; GINTHER, O.J. Follicular populations during the estrous cycle in heifers. I. Influence of day. Animal Reproduction Science, v.26, n.1-4, p.649-659, 1987.

RHODES, F.M.; FITZPATRICK, L.A.; ENTWISTLE, K.W. et al. Sequential changes in ovarian follicular dynamics in Bos indicus heifers before and after nutritional anoestrus. Journal of Reproduction and Fertility, v.104, n.1, p.41-49, 1995.

SAVIO, J.D.; KEENAN, L.; BOLAND, M.P. et al. Pattern of growth of dominant follicles during the oestrous cycle of heifers. Journal of Reproduction and Fertility, v.83, n.2, p.663-671, 1988.

TAYLOR, C.; RAJAMAHENDRAN, R. Follicular dynamics, corpus luteum growth and regression in lactating dairy cattle. Canadian Journal of Animal Science, v.71, n.3-4, p.61-68, 1991.

THIBIER, M. The animal embryo transfer industry in figures: a report from the IETS Data Retrieval Committee. IETS Newsletter, v.19, n.4, p.16-22, 2001.

UNIVERSIDADE FEDERAL DE VIÇOSA-UFV. SAEG -Sistema de análises estatísticas e genéticas. Versão 7.1. Viçosa:, MG, 1997. 150p. (Manual do usuário).

VIANA, J.H.M.; TORRES, C.A.A.; FERNANDES, C.A.C. et al. Ovarian follicular population and superovulatory response in beef cattle. Brazilian Journal of Morphological Sciences, v.16, n.2, p.175-179, 1999.

ZEITOUN, M.M.; RODRIGUEZ, H.F.; RANDEL, R.D. Effect of season on ovarian follicular dynamics in Brahman cows. Theriogenology, v.45, n.8, p.1577-1581, 1996.

Recebido em: 17/12/02

Aceito em: 22/04/04 